\title{
DETERMINACIÓN DE LEPTINA Y SUS VALORES SÉRICOS EN ALPACAS HEMBRAS ADULTAS CON DIFERENTE CONDICIÓN CORPORAL
}

\section{Leptin Determination and Serum Levels in Adult Alpacas with Different Body Condition}

\author{
Marco Enciso H., ${ }^{1,2}$, Raquel Pérez-Clariget ${ }^{3}$, Héctor Huamán U. ${ }^{4}$, \\ Óscar Cárdenas M. ${ }^{5}$ y Wilfredo Huanca L. ${ }^{1}$
}

\section{Resumen}

\begin{abstract}
El presente estudio tuvo como objetivo determinar la presencia de la hormona leptina en alpacas adultas, cuantificar sus valores y relacionarlos con la condición corporal. Se utilizaron 36 alpacas hembras adultas, vacías y sin cría, las cuales fueron divididas en dos grupos, según su condición corporal (CC): G1: CC <3.0 y G2: CC >3.0, de acuerdo a una escala 1 - 5 (1: emaciada, 5: obesa). Se tomaron muestras de sangre por punción de la vena yugular y el suero resultante fue mantenido en congelación a $-20^{\circ} \mathrm{C}$ hasta su análisis. La determinación de leptina fue realizada mediante la técnica de radioinmunoensayo (RIA). La media general de la concentración de leptina fue de $17.23 \pm 0.81 \mathrm{ng} / \mathrm{ml}$. Los valores encontrados para G1 fueron de $18.14 \pm 1.12 \mathrm{ng} / \mathrm{ml}$ y para $\mathrm{G} 2: 16.32 \pm 1.15 \mathrm{ng} / \mathrm{ml}$, sin diferencias estadísticas entre grupos. Los resultados obtenidos permiten evidenciar la presencia de leptina en alpacas.
\end{abstract}

Palabras clave: alpaca, leptina, condición corporal, radioinmunoensayo

\section{Abstract}

The aim of this study was to determine the presence of leptin in adults alpacas, in order to quantify leptin values for these animals, and to relate them with body condition. For this purpose, 36 non pregnant and non nursing female adult alpacas were used. They were divided in two groups according to body condition (BC): G1: BC <3.0 and G2: $\mathrm{BC}>3.0$, using the 1 to 5 scale (1: emaciate, 5: obese). Blood samples by jugular venipunction to obtain serum were taken. Serum samples were maintained at $-20^{\circ} \mathrm{C}$ until analysis. The leptin determination was carried out by the radioimmunoassay technique (RIA). The mean of leptin concentration was $17.23 \pm 0.81 \mathrm{ng} / \mathrm{ml}$. Values for G1 were $18.14 \pm 1.12 \mathrm{ng} /$ $\mathrm{ml}$ and for $\mathrm{G} 2$. were $16.32 \pm 1.15 \mathrm{ng} / \mathrm{ml}$, without statistical differences between groups. The results showed that the leptin hormone is also present in alpacas.

Key words: alpaca, leptin, body condition, radioimmunoassay

\footnotetext{
${ }^{1}$ Laboratorio de Reproducción Animal, ${ }^{4}$ Laboratorio de Bioquímica, Nutrición y Alimentación Animal, Facultad de Medicina Veterinaria, Universidad Nacional Mayor de San Marcos, Lima

${ }^{2}$ E-mail: marco.enciso@gmail.com

${ }^{3}$ Facultad de Agronomía, Universidad de la República, Uruguay

${ }^{5}$ Estación Experimental ILLPA, INIEA, Puno
} 
La ganadería alpaquera constituye una actividad pecuaria de gran importancia económica para los pobladores de las zonas altoandinas del país (Fernández Baca, 1971). En la alpaca, la eficiencia reproductiva es un componente importante en su sistema productivo; sin embargo, su nivel de productividad se ve drásticamente afectado por la baja eficiencia reproductiva que se presenta en su ambiente de crianza, la cual, expresada en tasa de natalidad, no es mayor al 50\% (Novoa, 1991). En tal sentido, la identificación, estudio, y entendimiento de los factores que afectan la reproducción en alpacas son metas importantes para la sostenibilidad a largo plazo de la crianza alpaquera.

La nutrición es uno de los principales componentes que influye en la reproducción. Los nutrientes son distribuidos hacia todo el organismo para mantener la homeostasis y los sobrantes son utilizados para el crecimiento y reproducción (Wade et al., 1996). Como existen demandas nutricionales para los tejidos y producción láctea, las funciones reproductivas se ven comprometidas si es que no se logra una compensación en la toma de nutrientes; por consiguiente, la pubertad y el reinicio de la actividad reproductiva post parto pueden tardarse (Butler, 2005).

Los mecanismos fisiológicos a través de los cuales la nutrición media sus efectos sobre la reproducción aún no han sido bien caracterizados. Una variedad de compuestos metabólicos, neurotransmisores y hormonas han sido implicados en la comunicación entre el estado nutricional y los centros que controlan la reproducción (Wade et al.,1996). Evidencias sugieren que la leptina, una hormona proteica sintetizada principalmente por el tejido adiposo, juega un rol importante en esta red de comunicación (Friedman y Halaas, 1998).
La leptina es una proteína de $16 \mathrm{kDa}$ producida por el gen de la obesidad $(o b)$. Está formada por 167 aminoácidos colocados en cuatro hélices alfa. El nombre de la leptina proviene del griego leptos, que significa "delgado" (Friedman y Halaas, 1998). Fue descubierta en 1994 y se le consideró como la hormona de la anti-obesidad (Zhang et al., 1994). Por primera vez en la historia, la simple administración de una proteína a un ratón genéticamente obeso, redujo la cantidad de grasa y masa corporal y aumentó los niveles de energía (Pelleymounter et al., 1995).

El ARNm de leptina ha sido aislado del cerebro, hipotálamo, glándula pituitaria (Morash et al., 1999; Wiesner et al., 1999), músculo esquelético (Wang et al., 1998), epitelio del estómago (Bado et al., 1998), placenta (Hoggard et al., 1997), ovarios, glándula mamaria y hueso (Margetic et al., 2002), pero la mayor producción de esta hormona ocurre en el tejido adiposo (Houseknecht $e t$ al., 1998).

Las concentraciones plasmáticas de leptina reflejan la cantidad de tejido adiposo en el cuerpo y tienden a aumentar y disminuir con ganancia y pérdida de peso, respectivamente. En animales normales, la leptina actúa como una señal aferente al sistema nervioso central (SNC) indicando que el cuerpo está en equilibrio energético positivo o negativo. Esto causa un cambio apropiado en el apetito, como una forma de corregir el desequilibrio y mantener peso corporal. Sin embargo, los ratones deficientes de leptina ( $o b /$ $o b)$ son hiperfágicos y masivamente obesos debido a su incapacidad para regular el apetito. Estas patologías pueden ser corregidas por administración de leptina exógena (Pelleymounter et al., 1995).

En rumiantes domésticos como bovinos y ovinos, la leptina interviene significativamente en la regulación del eje reproductivo y en la disponibilidad y metabolismo de energía (Chilliard et al., 2005). En alpacas no se cuen- 
ta con información sobre la presencia de leptina. Por eso, el presente trabajo pretendió evidenciar la presencia de la hormona leptina en alpacas adultas, cuantificar valores de leptina para estos animales, y relacionarlos con la condición corporal, indicativo del estado nutricional.

\section{Materiales y Métodos}

\section{Lugar de estudio}

El trabajo se llevó a cabo en el Centro de Investigación y Producción Quimsachata, de la Estación Experimental Illpa, Instituto Nacional de Investigación y Extensión Agraria (INIEA) - Puno. La estación se ubica a una altitud de 4,200 msnm, en el distrito de Santa Lucía, provincia de Lampa, departamento de Puno, en una zona agroecológica de puna seca, piso altitudinal sub alpino, cuyo clima es de tipo semi-seco frío, siendo la temperatura media de $7{ }^{\circ} \mathrm{C}$ (máxima $18^{\circ} \mathrm{C}$ y mínima $-13{ }^{\circ} \mathrm{C}$ ) con $485 \mathrm{~mm}$ de precipitación pluvial anual.

\section{Manejo de los animales}

Se utilizaron 36 alpacas hembras adultas mayores de tres años, no gestantes, sin cría al pie y con, por lo menos, un parto en su historial reproductivo. Los animales realizaban un pastoreo extensivo en su vegetación natural, la cual estaba conformada mayoritariamente por gramíneas, ciperáceas, juncáceas y leguminosas. La salida de los animales a la zona de pastoreo se iniciaba a las 06:00 y el retorno al corral de encierro era alrededor de las 17:00 a 18:00, con un periodo de ayuno en el encierro de, aproximadamente, $12 \mathrm{~h}$.

Las alpacas fueron seleccionadas al azar y distribuidas en dos grupos: $\mathrm{G} 1=18$ alpacas con condición corporal <3.0, y G2 = 18 alpacas con condición corporal >3.0.

\section{Condición corporal y toma de muestras}

A todos los animales se les midió la condición corporal, de acuerdo a una escala subjetiva de 1 a 5 , donde 1 : emaciada y 5 : obesa (Huanca et al., 1998). La técnica consistió en la palpación de la zona lumbar, pelviana, base del cuello y base de la cola; determinándose posteriormente, que las dos primeras (zona lumbar y pelviana) eran las más apropiadas, debido a que en éstas se detectaban mayores diferencias del estado nutricional.

Se tomaron muestras de sangre temprano en la mañana, con los animales en ayuno, antes de que salgan a pastoreo. Se colectó 5 a $7 \mathrm{ml}$ de sangre por punción de la vena yugular. Las muestras fueron centrifugadas a $4000 \mathrm{rpm}$ por espacio de siete minutos. El suero resultante fue almacenado en viales a $-20{ }^{\circ} \mathrm{C}$ hasta su análisis.

\section{Determinación de leptina}

La concentración de leptina se determinó mediante la técnica de radioinmunoensayo (RIA) específico para ovinos descrito por Delavaud et al. (2000). El procedimiento fue realizado en el Laboratorio de Veterinaria del Centro de Investigaciones Nucleares (CIN), Facultad de Ciencias, Universidad de la República, Montevideo, Uruguay. El RIA es de doble anticuerpo y utiliza leptina recombinante ovina con $98 \%$ de pureza de acuerdo a SDS-PAGE, y marcada con $\mathrm{I}^{125}$. El anticuerpo es producido en conejos. El RIA utilizado ha sido validado en humanos, en varias especies domésticas tales como ovinos, bovinos, caprinos, llamas, pollos, ratas, ratones y perros, y en algunas especies silvestres como ciervo elk (Cervus elaphus), reno (Rangifer tarandus), alce (Alces alces), elefante africano (Loxodonta africana) y elefante marino del sur (Mirounga leonina). En la validación se utilizó suero sanguíneo, plasma sanguíneo, le- 
che y líquido céfalo raquídeo, observándose en todos los casos buenas curvas de paralelismo (Duane H. Keisler, comunicación personal; Guilherme et al., 2004). Antes de analizar las muestras, se corrieron las correspondientes curvas de paralelismo para suero de alpaca.

Todas las muestras del presente estudio fueron analizadas en un solo ensayo por triplicado. El límite de detección fue $0.1 \mathrm{ng} /$ $\mathrm{ml}$ y el coeficiente de variación intraensayo fue de $10 \%$.

\section{Análisis estadístico}

Se utilizó la prueba de «t» de Student de Independencia para analizar la diferencia entre la media de los valores de leptina entre G1 y G2. El nivel de significancia empleado para el análisis de los resultados fue de 5\%. Los datos obtenidos fueron analizados utilizando el paquete estadístico GraphPad Prism ${ }^{\circledR}$ 3.0 (GraphPad Software, 1999).

\section{Resultados}

La media general de la concentración de leptina fue de $17.23 \pm 0.81 \mathrm{ng} / \mathrm{ml}$. Los valores séricos de leptina en los animales con condición corporal $<3$ y $>3$ fueron de $18.14 \pm$ 1.12 y $16.32 \pm 1.15 \mathrm{mg} / \mathrm{ml}$, respectivamente, sin que hubiera diferencias significativas.

Los valores extremos de la concentración de leptina en general fueron de 10.17 ng/ml (mínimo) a 28.39 ng/ml (máximo), sin haber diferencia entre los mínimos y máximos para G1 y G2.

\section{Discusión}

Esta es la primera vez que se obtienen datos de valores séricos de leptina en alpacas, lo que permite confirmar la presencia de la hormona en esta especie de camélido, así como disponer de una estimación de sus valores. La literatura internacional señala que la leptina se encuentra presente en muchos vertebrados, especialmente mamíferos y aves (Friedman y Halaas, 1998; Dridi et al., 2000) e incluso reptiles (Lewis, 2000; Paolucci et al., 2001); sin embargo, no se había evidenciado su presencia en alpacas.

Los valores de leptina varían de acuerdo a la técnica que se emplea para su determinación. Se ha desarrollado protocolos para la medición de leptina con la técnica de RIA y con la técnica de ELISA (Hardie et al., 1996; Chow y Phoon, 2003). En ambas técnicas se obtiene una buena sensibilidad y especificidad a leptina, siendo el RIA la técnica con mayor difusión.

En el caso del método de RIA, existe un kit comercial llamado "multiespecie" (XL85K, Linco Research, St. Louis, MO, Estados Unidos), y RIA específicos no comerciales desarrollados para ovinos (Delavaud et al., 2000), bovinos (Ehrhardt et al., 2005) у pollos (Dridi et al., 2000). Sin embargo, el kit comercial de RIA multiespecie parece subestimar las concentraciones de leptina; por ejemplo, en muestras de ovinos y bovinos, dieron valores de $2.4 \pm 0.9 \mathrm{ng} / \mathrm{ml}$ y $2.2 \mathrm{ng} / \mathrm{ml}$ en tanto que se obtuvo valores de $7.5 \pm 3.3$ $\mathrm{ng} / \mathrm{ml}$ y $6.6 \mathrm{ng} / \mathrm{ml}$ al cuantificarse con el RIA específico, respectivamente (Delavaud et al., 2000, 2002). En tal sentido, al haber utilizado un RIA específico para rumiantes, el valor obtenido como media general para alpacas en el presente trabajo sería representativo $(17.23 \pm 0.81 \mathrm{ng} / \mathrm{ml})$ de la especie. Además, es importante resaltar que los valores de leptina en alpacas fueron mucho más altos que los observados en otros rumiantes domésticos adultos, siendo la cabra la única especie que presenta valores de leptina superiores a los $10 \mathrm{ng} / \mathrm{ml}$ (Bonnet et al., 2005).

La concentración de leptina varía de acuerdo a la edad, como ha sido demostrado en caballos (Buff et al., 2002), bovinos (Blum et al., 2005), ovinos (Äebulj-Kadunc y 
Cestnik, 2005) y en humanos (Chow y Phoon, 2003). No se podría determinar si este factor ha influido en los resultados del presente trabajo, ya que las hembras utilizadas fueron adultas (entre 3 y 9 años de edad); por lo que sería necesario determinar los niveles de leptina en alpacas menores de 2 ó mayores de 10 años.

Los niveles de leptina están correlacionados positivamente con la condición corporal (CC) y el contenido graso (Delavaud et al., 2000; Buff et al., 2002), aspecto que no pudo ser corroborado en el presente estudio. De hecho, los valores mínimos y máximos obtenidos en alpacas con $\mathrm{CC}<3.0 \mathrm{y}>3.0$ son similares (mínimos: $10.17 \mathrm{ng} / \mathrm{ml}$ y $10.48 \mathrm{ng}$ / $\mathrm{ml}$ y máximos $26.74 \mathrm{ng} / \mathrm{ml}$ y $28.39 \mathrm{ng} / \mathrm{ml}$, respectivamente). Es decir en ambos grupos la concentración de leptina varió alrededor de 62 a $63 \%$.

Cabe resaltar que los grados de $\mathrm{CC}$ en las alpacas no son tan evidentes como ocurre en otras especies domésticas, y que en el caso de llamas se está intentando estandarizar la técnica de CC (Rigalt et al., 2006). Por otro lado, las alpacas con alimentación en base a pasturas naturales tienden a mantener una $\mathrm{CC}$ uniforme.

\section{Conclusiones}

? Se confirma la presencia de la hormona leptina en alpacas adultas vacías.

? Los niveles séricos de leptina obtenidos son más elevados que aquellos en otros rumiantes domésticos adultos.

? No se observaron diferencias en la concentración de leptina en alpacas con condición corporal $<3.0$ y $>3.0$.

\section{Literatura Citada}

1. Bado A, Levasseur $S$, Attoub $S$, Kermorgant $S$, Laigneau JP, Bortoluzzi MN, Moizo L, Lehy T,
Guerre-Millo M, Le MarchandBrustel L, Lewin MJM. 1998. The stomach is a source of leptin. Nature 394: 790-793.

2. Blum JW, Zbinden Y, Hammon HM, Chilliard Y. 2005. Plasma leptin status in young calves: effects of pre-term birth, age, glucocorticoid status, suckling, and feeding with and automatic feeder or by bucket. Dom Anim Endocrinol 28: 119133.

3. Bonnet M, Delavaud C, Rouel J, Chilliard Y. 2005. Pregnancy increases plasma leptin in nulliparous but not primiparous goats while lactation depresses it. Dom Anim Endocrinol 28: 216-223.

4. Buff PR, Dodds AC, Morrison CD, Whitley NC, McFadin EL, Daniel JA, Djiane J, Keisler DH. 2002. Leptin in horses: Tissue localization and relationship between peripheral concentrations of leptin and body condition. J Anim Sci 80: 2942-2948.

5. Butler WR. 2005. Relationships of negative energy balance with fertility. Adv Dairy Tech 17: 35-46.

6. Äebulj-Kadunc N,Cestnik V. 2005. Circulating leptin concentrations in Lipizzan horses and Jezersko-Solchava sheep. Slov Vet Res 42(1-2): 11-14.

7. Chilliard Y, Delavaud C, Bonnet M. 2005. Leptín expression in ruminants: Nutritional and physiological regulations in relation with energy metabolismo. Dom Anim Endocrinol 29: 3-22.

8. Chow VTK. Phoon MC. 2003. Measurement of serum leptin concentrations in university undergraduates by competitive ELISA reveals correlations with body mass index and sex. Adv Physiol Ed 27: 70-77.

9. Delavaud C, Ferlay A, Faulconnier $Y$, Bocquier F, Kann G, Chilliard Y. 2002. Plasma leptin concentration in adult cattle: Effects of breed, adiposity, feeding level, and meal intake. J Anim Sci 80: 1317-1328. 
10. Delavaud C, Bocquier F, Chilliard Y, Keisler DH, Gertler A. 2000. Plasma leptin determination in ruminants: effect of nutritional status and body fatness on plasma leptin concentration assessed by a specific RIA in sheep. J Endocrinol 165: 519-526.

11. Dridi S, Williams J, Bruggeman V, Onagbesan M, Raver N, Decuypere E, Djiane J, Gertler A, Taouis M. 2000. A chicken leptin-specific radioinmmunoassay. Dom Anim Endocrinol 18: 325-335.

12. Ehrhardt RA, Slepetis RM, SiegalWillott J, van Amburgh ME, Bell AW, Boisclair YR. 2000. Development of a specific radioimmunoassay to measure physiological changes of circulating leptin in cattle and sheep. J Endocrinol 166: 519528.

13. Fernández Baca S. 1971. La alpaca: reproducción y crianza. Boletín de Divulgación $\mathrm{N}^{\mathrm{o}} 7$. Centro de Investigación IVITA, UNMSM. Lima. 43 p.

14. Friedman JM, Halaas JL. 1998. Leptin and the regulation of body weight in mammals. Nature. 395: 763-770.

15. Guilherme C, Bianchini A, Martinez RB, Robaldo RB, Colares EP. 2004. Serum leptin concentration during the terrestrial phase of the Southern elephant seal Mirounga leonina (Carnivora: Phocidae). Gen Comp Endocrinol 139: 137-142.

16. Hoggard N, HunterL, DuncanJS, Williams LM, Trayhurn P, Mercer JG. 1997. Leptin and leptin receptor mRNA and protein expression in the murine fetus and placenta. Proc Natl Acad Sci,USA 94: 11073-11078.

17. Houseknecht KL, Baile CA, Matteri RL, Spurlock ME. 1998. The biology of leptin: A review. J Anim Sci 76: 14051420.

18. Huanca W, Camacho J, Cordero A, Ampuero A, Santiago B, Quiñónez C. 1998. Evaluación clínica testicular y biometría de alpacas machos en la sierra central. Res XXI Reunión Científica Anual APPA. Puno, Perú. p 167-169.
19. Hardie LJ, Rayner DV, Holmes S, Trayhurn P. 1996. Circulating leptin levels are modulated by fasting, cold exposure, and insulin administration in lean but not Zucker $(f a / f a)$ rats as measured by ELISA. Biochem Biophys Res. Comm 223: 660-665.

20. Lewis R. 2000. Leptin lizards. Hormone's discovery in reptiles suggests role in energy allocation. The Scientist 14(3): 20.

21. Margetic S, Gazzola C, Pegg GG, Hill RA. 2002. Leptin: A review of its peripherial actions and interactions. Int J Obesity 26: 1407-1433.

22. Morash B, Li A, Murphy PR, Wilkinson M, Ur E. 1999. Leptin gene expression in the brain and pituitary gland. Endocrinology 140:5995-5998.

23. Novoa C. 1991. Fisiología de la reproducción en la hembra. En: Fernández Baca S. (ed.) Avances y perspectivas del conocimiento de los camélidos sudamericanos. Santiado, Chile: FAO. Oficina Regional de la FAO para América Latina y el Caribe. p 91-109.

24. Paolucci M, Rocco M, Varricchio E. 2001. Leptin presence in plasma, liver and fat bodies in the lizad Podarcis sicula. Fluctuations throughout the reproductive cycle. Life Sci 69: 23992408.

25. Pelleymounter MA, Cullen MJ, Baker $M B$, Hecht R, Winters D. 1995. Effects of obese gene product on body weight regulation in ob/ob mice. Science 269: 540-543.

26. Rigalt F, Ferrando C, Pivotto R, Krapp C, Gomez R. 2006. Ajuste de la técnica de determinación de nota de condición corporal (NCC) en llamas (Lama glama). En: Olivera, D.; M. Miragaya, S. Piug (eds.) Resumenes IV Congreso Mundial sobre Camélidos. Santa María, Argentina. $p 48$.

27. Wade GN, Schneider JE, Li HY. 1996. Control of fertility by metabolic cues. Am J Physiol 270: E1-E19.

28. Wang J, Liu R, Hawkins M, Barzilai $N$, Rossetti L. 1998. A nutrient-sensing 
pathway regulates leptin gene expression in muscle and fat. Nature 393: 684-688.

29. Wiesner G Vaz M, Collier G, Seals D, Kaye D, Jennings G, Lambert $G$, Wilkinson D, Esler M. 1999. Leptin is released from the human brain: Influence of adiposity and gender. J Clin Endocrinol Metabol 84: 2270-2274.

30. Zhang Y, Proenca R, Maffei M, Barone M, Leopold L, Friedman JM. 1994. Positional cloning of the mouse obese gene and its human homologue. Nature 372: 425-432. 